\title{
Propiedades psicométricas del Exercise Addiction Inventory (EAI) en una muestra de estudiantes brasileños universitarios*
}

\section{Psychometric properties of the Exercise Addiction Inventory (EAI) in} a sample of brazilian university students

Recepción: 06 Junio 2014 | Aprobación: 24 Enero 2017

\author{
Álvaro Sicilia ${ }^{\mathrm{a}}$ \\ Universidad de Almería, España \\ ORCID: http://orcid.org/0000-0001-9436-8743 \\ VAlter Bracht \\ Universidade Federal do Espirito Santo, Brasil \\ Vinicius Penha \\ Universidade Federal do Espirito Santo, Brasil \\ Ueberson Ribero Almeida \\ Universidade Federal do Espirito Santo, Brasil \\ ROBERTO FERriz \\ Universidad de Almería, España \\ Manuel Alcaraz-Ibáñez \\ Universidad de Almería, España
}

a Autor de correspondencia. Correo electrónico: asicilia@ual.es

Para citar este artículo: Sicilia, A., Bracht, V., Penha, V., Ribero, U., Ferriz., R., \& AlcarazIbañez, M. (2017). Propiedades psicométricas del Exercise Addiction Inventory (EAI) en una muestra de estudiantes brasileños universitarios. Universitas Psychologica, 16(2), 1-10.

https://doi.org/10.11144/Javeriana.upsy16-2.ppea

\section{RESUMEN}

El objetivo de este estudio fue validar el Inventario de Adicción al Ejercicio (EAI) en el contexto brasileño. Participaron en el estudio 251 estudiantes universitarios (hombres $=132$, mujeres $=119$, Medad $=$ 22.34 , DT $=4.65)$. Los resultados mostraron un adecuado ajuste del modelo a los datos: $\chi^{2}(9, \mathrm{~N}=251)=17.45, \mathrm{p}=0.04 ; \chi^{2} / \mathrm{gl}=1.94$; $\mathrm{CFI}=0.96 ; \mathrm{IFI}=0.96 ; \mathrm{RMSEA}=0.061(\mathrm{IC} 90 \%=0.01-0.10)$; $\mathrm{SRMR}=0.041$. La estructura factorial se mostró invariante respecto del género. Se obtuvieron adecuados valores de consistencia interna $(p=0.70)$ y estabilidad temporal $(\mathrm{CCI}=0.80)$. Dieciséis $(6.4 \%)$ participantes fueron clasificados en riesgo de adicción, 198 (78.9\%) como sintomáticos y $37(14.7 \%)$ como asintomáticos. Se obtuvieron evidencias que permiten sugerir la validez y la fiabilidad del EAI en el contexto brasileño. No obstante, las investigaciones con poblaciones más diversas deberían determinar la aplicabilidad general del EAI.

Palabras clave

adicción; ejercicio físico; psicometría; validez

\begin{abstract}
The purpose of this study was to validate the Exercise Addiction Inventory (EAI) in the Brazilian context. A total of 251 university students (male $=132$, female $=119$, Mage $=22.34, \mathrm{SD}=4.65)$ participated in the study. Results showed an adequate fit to the data: $\chi^{2}(9, \mathrm{~N}=251)=$ $17.45, \mathrm{p}=.04 ; \chi 2 / \mathrm{gl}=1.94 ; \mathrm{CFI}=.96 ; \mathrm{IFI}=.96 ; \mathrm{RMSEA}=.061(\mathrm{IC}$ $90 \%=.01-.10) ;$ SRMR $=.041$. The factorial structure was invariant across gender. Adequate values of internal consistency $(p=.70)$ and temporal stability $(\mathrm{ICC}=.82)$ were obtained. Sixteen students $(6.4 \%)$
\end{abstract}


were classified at risk of exercise addiction, 198 (78.9 $\%)$ as symptomatic and $37(14.7 \%)$ as asymptomatic. Evidence for the reliability and validity of the EAI in the Brazilian context were obtained. Nevertheless, research in more diverse populations is needed to further examine the general applicability of the EAI.

Keywords

addiction; exercise; psychometrics; validity of test

El concepto de adicción ha estado en el pasado asociado a la ingestión de drogas. No obstante, más recientemente, algunas conductas (e. g., juego, sexo, uso de internet, ejercicio físico) han sido consideradas potencialmente adictivas (Sussman, Lisha, \& Griffiths, 2011). En esta línea, la clasificación recogida en el Diagnostic and Statistical Manual of Mental Disorders (DSM-V; American Psychiatric Association, 2010) sitúa los desórdenes conductuales en el mismo apartado que los desórdenes adictivos relacionados con el consumo de sustancias. Esta nueva clasificación parece basarse en las recientes evidencias que sugieren que la adicción podría reflejar un desorden en el sistema de recompensa del cerebro, independiente de si dicho sistema es activado por una conducta o una sustancia (Grant, Potenza, Weinstein, \& Gorelick, 2010). No obstante, aunque el DSM-V solo recoge como adicción de carácter conductual y de forma específica la adicción al juego, la nueva conceptualización sobre el diagnóstico de la adicción plantea la necesidad de investigar sobre otras formas de conducta excesiva y potencialmente adictivas, como podría ser el caso del ejercicio físico.

La literatura muestra que para referirse a la adicción al ejercicio se han utilizado términos como dependencia, compromiso, ejercicio obligatorio, ejercicio abusivo y ejercicio excesivo (Farrell \& Thompson, 1998; Hausenblas \& Symons-Downs, 2002b; Kagan, 1987). Aunque en este artículo se utilizará el término adicción al ejercicio en línea con la clasificación establecida por el DSM-V, en general, los términos anteriormente aludidos hacen referencia a la condición por la cual la práctica de actividad física adquiere rasgos adictivos. En concreto, dicha conducta se caracteriza por la presencia de un fuerte deseo de realizar ejercicio y que puede conducir a la aparición de síntomas fisiológicos (e. g., tolerancia, abstinencia) o psicológicos (e. g., ansiedad, depresión) (Hausenblas \& Symons-Downs, 2002b, 2002c). No obstante, la investigación realizada en torno a este desorden se ha mostrado ambigua, dado que hasta el momento no se ha alcanzado un acuerdo acerca de la caracterización del constructo y, por extensión, de las medidas que permitirían evaluarlo.

Desde que Glasser (1976) usó el término adicción positiva para referirse a los aspectos beneficiosos del ejercicio físico y diferenciarlo del carácter autodestructivo de conductas como el consumo abusivo de drogas, ha existido un creciente interés en intentar diferenciar los aspectos positivos y negativos de la práctica de ejercicio físico. Advirtiendo del peligro de identificar el carácter excesivo con el adictivo en este contexto, Morgan (1979) reconoció la existencia de posibles efectos negativos asociados a dicha conducta. En esta línea, Szabo, Frenkl y Caputo (1997) advirtieron que la literatura podría recoger un amplio número de estudios sobre adicción al ejercicio que en realidad estarían reflejando un elevado nivel de compromiso con esta conducta. En cualquier caso, establecer conceptualmente cuándo la existencia de un elevado compromiso con la práctica de ejercicio físico adquiere la condición de conducta adictiva se muestra como una tarea compleja (Johnston, Reilly, \& Kremer, 2011).

Esta escasa concreción conceptual y la subsiguiente falta de precisión a la hora de delimitar la adicción al ejercicio podrían haber dificultado la tarea de obtener una estimación exacta de su prevalencia. Así, mientras que un amplio número de estudios indica que la adicción al ejercicio es bastante reducida en la población general (Griffiths, Szabo, \& Terry, 2005; Mónok et al., 2012; Symons-Downs, Hausenblas, \& Nigg, 2004), otros han observado elevados niveles de prevalencia en poblaciones específicas de triatletas y usuarios de centros deportivos (Blaydon \& Lindner, 2002; Lejoyeux, Avril, Richoux, Embouazza, \& Nivoli, 2008). Pese a que esta disparidad en las estimaciones 
pudiera deberse a las diferentes características de las poblaciones de estudio, esta también podría venir provocada por los instrumentos utilizados para medir el constructo.

Los primeros instrumentos diseñados para medir la adicción al ejercicio, entre los cuales se encontraban el Commitment to Running Scale (Carmack \& Martens, 1979) y la Negative Addiction Scale (Hailey \& Bailey, 1982) fueron medidas unidimensionales que evaluaban tan solo determinados aspectos del fenómeno (Hausenblas \& Symons-Downs, 2002b). Posteriormente, se realizaron aproximaciones multidimensionales que comprendían un conjunto variado de síntomas (Freimuth, Moniz, \& Kim, 2011; Hausenblas \& Symons-Downs, 2002c). En esta línea, Davis, Brewer y Ratusny (1993) desarrollaron el Commitment to Exercise Scale (CES), instrumento que evaluaba un aspecto obligatorio (e. g., sentirse culpable cuando se pierde una sesión de entrenamiento) y un aspecto patológico (e. g., continuar realizando ejercicio a pesar de estar enfermo o lesionado) del constructo. Más tarde, Ogden, Veale y Summers (1997) desarrollaron el Exercise Dependence Questionnaire (EDQ), el cual recogía ocho dimensiones basadas conceptualmente en los criterios clínicos de la dependencia de sustancias recogidos en el DSM-IV (American Psychiatric Association, 1994). No obstante, este tipo de medidas fueron creadas sin contemplar un criterio de corte que contribuyese a detectar posibles casos de adicción al ejercicio.

Para solventar esta limitación, Hausenblas y Symons Downs (2002a, 2002c) desarrollaron la Exercise Dependence Scale (EDS), siendo este el primer instrumento que conceptualizó e instrumentalizó la adicción al ejercicio basándose en los siete síntomas recogidos en el DSMIV (i. e., abstinencia, continuación, tolerancia, falta de control, reducción de otras actividades, tiempo empleado y efectos deseados). Tras una posterior revisión (Symons-Downs et al., 2004), la versión final del instrumento quedó formada por 21 ítems agrupados a razón de 3 para cada uno de los 7 síntomas anteriormente reseñados y que a través de cálculos indirectos permite diferenciar a los sujetos como en riesgo de adicción, sintomáticos y asintomáticos. No obstante, pese a que la EDS se diseñó partiendo de una sólida base teórica, lo cierto es que en función del tiempo requerido para su administración y de la dificultad presente en los cálculos necesarios para realizar la clasificación, el instrumento se muestra poco práctico para su uso cotidiano por parte de los profesionales (e. g. médicos, psicólogos, fisioterapeutas, monitores o entrenadores deportivos). Atendiendo a esta limitación, Terry, Szabo y Griffiths (2004) desarrollaron el Exercise Addiction Inventory (EAI), una herramienta que permitía medir la adicción al ejercicio de forma breve y práctica.

El EAI es un instrumento que, en lugar de basarse en criterios percibidos por el investigador (e. g., CES, EDQ) o en aquellos propios de la dependencia de sustancias (e. g., EDS), se basa en los criterios definidos por las teorías específicas de las adicciones de carácter conductual, situándose, por tanto, más en línea con la nueva clasificación recogida en el DSMV. De esta forma, los síntomas de adicción al ejercicio son implementados a través de seis ítems que recogen cada uno de los componentes de la adicción conductual definidos por Griffiths (1996): 1) relevancia: cuando el ejercicio físico llega a ser la actividad más importante en la vida de la persona alcanzando a dominar otras facetas de su vida; 2) euforia: experiencia subjetiva que la persona manifiesta como consecuencia de realizar ejercicio; 3) tolerancia: la persona tiende a incrementar la cantidad de ejercicio realizado para conseguir los efectos deseados; 4) abstinencia: sentimiento o efecto físico desagradable producido cuando la realización de ejercicio es interrumpida o drásticamente reducida; 5) conflicto: tensión que puede producirse entre la conducta adoptada al realizar ejercicio y aquellos que rodean a la persona (e. g., conflicto interpersonal) o dentro de la misma persona (e. g., conflicto intrapsíquico); f) recaída: tendencia a repetir los mismos patrones de ejercicio, o aún más acentuados, después de un tiempo sin realizar la actividad o una retirada por lesión. Atendiendo a la puntuación total de sus ítems, el EAI opera como una herramienta de cribado que permite distinguir 
entre sujetos en riesgo de adicción al ejercicio, sintomáticos o asintomáticos. Utilizando una muestra de estudiantes universitarios, Terry et al. (2004) aportaron evidencias acerca de las adecuadas propiedades psicométricas del EAI, apreciando una alta correlación con otros instrumentos que miden el mismo constructo. Además, la fiabilidad del instrumento fue probada con una muestra independiente (Griffiths et al., 2005). Posteriormente, el EAI ha sido adaptado y validado a distintos idiomas, habiendo mostrado adecuadas características psicométricas en estudios realizados en países como Dinamarca, Hungría, España, Reino Unido o Estados Unidos (Griffiths et al., 2015).

El presente estudio tuvo como objetivo analizar las propiedades psicométricas del Exercise Addiction Inventory (Terry et al., 2004) al contexto brasileño. Se espera que, en línea con anteriores trabajos, el EAI presente una estructura unifactorial formada por seis ítems y que permanezca invariante en función del género. De igual forma, se espera que el instrumento muestre adecuados niveles de consistencia interna y estabilidad temporal.

\section{Método}

\section{Participantes}

Participaron en este estudio 251 estudiantes (132 hombres y 119 mujeres), con edades comprendidas entre 17 y 46 años $(\mathrm{M}=$ 22.34; $\mathrm{DT}=4.65)$, pertenecientes a una universidad del sudeste de Brasil. La mayoría de los participantes $(96.4 \%)$ eran estudiantes de Educación Física o Ciencias del Deporte. Todos indicaron realizar de forma habitual ejercicio físico en su tiempo libre. Para analizar la estabilidad temporal de la escala, se empleó una muestra independiente de 56 estudiantes universitarios con edades comprendidas entre 20 y 31 años $(M=25.5 ; D T=0.92)$ que completaron el instrumento en dos ocasiones, con un intervalo de 2 semanas entre la primera y la segunda toma de datos.

\section{Medidas}

\section{Inventario de adicción al ejercicio}

Se utilizó la versión adaptada al portugués del Exercise Addiction Inventory (EAI; Terry et al., 2004). Este instrumento contiene seis ítems (e. g., "O exercício físico é a coisa mais importante de minha vida"). La escala estaba precedida de la sentencia "Em que grau você concorda com as seguintes afirmações", indicando los participantes su respuesta a cada ítem por medio de una escala Likert que oscilaba de 1 (Discordo totalmente) a 5 (Concordo totalmente). Puntuaciones más altas revelan mayores síntomas de adicción al ejercicio. El instrumento establece puntuaciones de corte para clasificar a los respondientes en función de su nivel de adicción al ejercicio, definiendo a estos como en riesgo (i. e., 24 o más puntos), sintomáticos (i. e., entre 13 y 23 puntos) y asintomáticos (i. e., 12 o menos puntos).

\section{Procedimiento}

Siguiendo el método de traducción inversa (Hambleton \& Patsula, 1998), la escala original fue traducida al portugués por un grupo de traductores, traduciendo posteriormente otro grupo independiente la escala a su idioma original. La bondad de la traducción se juzgó atendiendo al grado de coincidencia con la versión original. La versión obtenida fue analizada por tres expertos (Lynn, 1986) en actividad física para garantizar que los ítems retuviesen el significado original y reflejasen el constructo que se pretendía medir.

Obtenida la versión final de la escala, se contactó con el profesorado y los estudiantes para informarles de los objetivos del estudio y solicitar su colaboración. Aquellos estudiantes que voluntariamente expresaron su consentimiento informado cumplimentaron de forma anónima el instrumento en presencia de un miembro del equipo de investigación, el cual estuvo a su disposición para resolver las dudas que pudieron surgir durante el proceso. El tiempo requerido 
para cumplimentar el cuestionario estuvo en torno a 12 minutos. Este estudio contó con la aprobación del comité ético de la Universidade Federal do Espirito Santo.

Análisis de datos

En primer lugar, se analizaron los estadísticos descriptivos y las correlaciones bivariadas entre los ítems, empleando en este segundo caso el coeficiente $r$ de Pearson. El tamaño del efecto se interpretó atendiendo a los puntos de corte sugeridos por Ferguson (2009). A continuación, se realizó un análisis factorial confirmatorio (AFC), en el que en la función de la distribución normal univariada (asimetría $<|1.41|$; curtosis < $|-1.18|)$ y multivariada (coeficiente de Mardia $=1.30$; razón crítica $=1.05)$ de los datos se utilizó el método de estimación de máxima verosimilitud (Byrne, 2013). Para juzgar la adecuación del modelo, se consideraron varios índices de ajuste: $\chi 2 / g 1$, CFI (Comparative Fit Index), TLI (Tucker Lewis Index), IFI (Incremental Fit Index), RMSEA (Root Mean Square Error of Approximation) y su intervalo de confianza (IC) a $90 \%$ y SRMR (Standardized Root Mean Square Residual). En general, valores inferiores a 3 para $\chi 2 / \mathrm{df}$, valores iguales o superiores a 0.95 para CFI, TLI e IFI, valores iguales o inferiores a 0.06 para RMSEA y 0.08 para SRMR fueron considerados como indicativos de un ajuste adecuado del modelo a los datos (Hu \& Bentler, 1999). A continuación, se analizó la invariancia factorial por género del modelo, considerando que la hipótesis nula de invariancia debía ser rechazada en presencia de diferencias estadísticamente significativas entre el modelo sin restricciones y los sucesivos modelos restringidos (Byrne, 2013). Finalmente, se comprobó la fiabilidad del instrumento mediante: 1) un análisis de consistencia interna, utilizando los coeficientes alfa de Cronbach $(\alpha)$ y de fiabilidad compuesta de Raykov ( $\rho$, Raykov, 2004) y 2) un análisis de estabilidad temporal utilizando el coeficiente de correlación intraclase (CCI) y su IC al $95 \%$. Valores de consistencia interna y de estabilidad temporal superiores a 0.70 fueron considerados como adecuados (Fleiss, 2011; Nunnally \& Bernstein, 1995).

\section{Resultados}

Estadísticos descriptivos, análisis correlacional y prevalencia de adicción al ejercicio

Como se muestra en la Tabla 1, las desviaciones típicas de los ítems fueron superiores a 1 y las medias se distribuyeron tanto por encima del punto medio de la escala (e. g., ítems 3, 4 y 6) como por debajo (e. g., ítems 1,2 y 5). Los valores del coeficiente de correlación de Pearson (r) obtenidos entre cada par de ítems sugirió la existencia de una asociación de carácter débil. En concreto, el valor de $\mathrm{r}$ se situó entre 0.10 y 0.41 , siendo dichas relaciones estadísticamente significativas $(\mathrm{p}<0.05)$, excepto en el caso de la correlación entre los ítems 2 y 6 ( $\mathrm{p}=$ 0.11). El nivel de asociación entre cada ítem y la puntuación total de la escala fue moderado, oscilando el valor de la correlación entre 0.53 y 0.70 ( $\mathrm{p}<0.01$ ). En su conjunto, los valores del análisis correlacional no sugirieron la existencia de solapamientos. Un $6.4 \%$ de los estudiantes fueron clasificados como en riesgo de adicción al ejercicio, 78.9 \% como sintomáticos y $14.7 \%$ como asintomáticos.

\section{TABLA 1}

Estadísticos descriptivos y correlaciones entre los items del EAI

\begin{tabular}{|c|c|c|c|c|c|c|c|c|c|c|c|}
\hline İtenss variable & $M$ & $D T$ & $g l$ & g: & 1 & 2 & 3 & 4 & 5 & 6 & 7 \\
\hline 1. ftem 1 & 2.97 & 1.10 & -0.17 & -0.49 & 7 & $0.19^{* 8}$ & $0.33^{* *}$ & $0.36^{* *}$ & $0.36^{* *}$ & $0.18^{4 *}$ & $0.63^{* *}$ \\
\hline 2 [tem2 & 1.78 & 1.12 & 1.41 & 1.09 & & & $0.29^{* *}$ & $0.17^{* 8}$ & $0.32^{* *}$ & 0.10 & $0.53^{* *}$ \\
\hline 3. Item 3 & 3.71 & 1.13 & -0.66 & -0.26 & & & & $0.35^{* 8}$ & $0.41^{* *}$ & $0.20^{* *}$ & $0.67^{\mathrm{k}}$ \\
\hline 4. $\operatorname{ttem} 4$ & 3.33 & 1.26 & -0.27 & -0.94 & & & & & $0.26^{* *}$ & $0.30^{* *}$ & $0.66^{* *}$ \\
\hline 5. Item $\}$ & 2.20 & 1.20 & 0.70 & -0.47 & & & & & & $0.30^{* *}$ & $0.70^{\mathrm{k*}}$ \\
\hline 6. Item 6 & 3.04 & 1.37 & -0.03 & -1.18 & & & & & & & $0.59^{\mathrm{kp}}$ \\
\hline 7. EAI & 2.84 & 0.75 & 0.21 & -0.23 & 1 & & & & & & \\
\hline
\end{tabular}

Nota: g1 = asimetría; g2 = curtosis; EAI = puntuación total de la escala. ${ }^{* *} \mathrm{p}<0.01$ Fuente: Elaboración propia.

Análisis factorial confirmatorio

Los índices de ajuste obtenidos para el modelo unidimensional de seis ítems fueron satisfactorios: $\chi^{2}(9, \mathrm{~N}=251)=17.45, \mathrm{p}=$ $0.042 ; \chi^{2} / \mathrm{gl}=1.94 ; \mathrm{CFI}=0.96$; IFI $=0.96$; 
RMSEA $=0.061($ IC $90 \%=0.01,0.10) ;$ SRMR $=0.041$. El tamaño del efecto de la cargas factoriales fue moderado, oscilando entre $0.40 \mathrm{y}$ $0.64(\mathrm{p}<0.001)$.

\section{Figura 1}

Análisis factorial confirmatorio del EAI. La elipse representa el factor y los rectángulos los items. Los coeficientes de correlación al cuadrado se muestran en los círculos pequeños

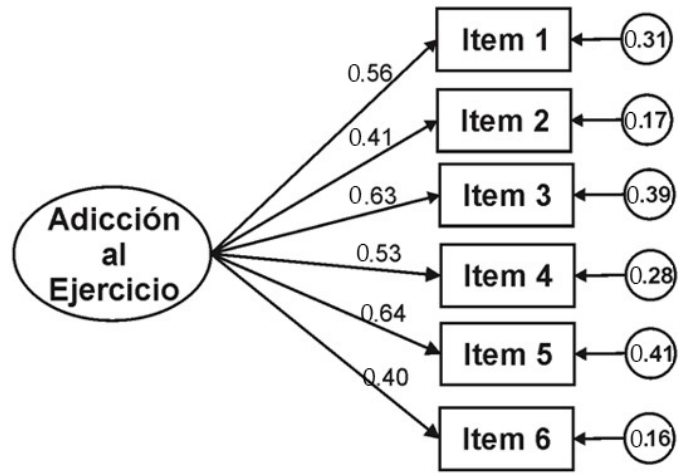

Fuente: elaboración propia.

Análisis de invariancia en función del género

Los cuatro modelos comparados mostraron buenos índices de ajuste (Tabla 2). No se apreciaron diferencias estadísticamente significativas entre el modelo sin restricciones (Modelo 1) y los modelos con pesos de medida invariantes (Modelo 2), covarianzas estructurales invariantes (Modelo 3) y residuos de medida invariantes (Modelo 4), no pudiendo rechazarse, por tanto la hipótesis nula de invariancia.

TABLA 2

Análisis multigrupo de invariancia en función del género

\begin{tabular}{|c|c|c|c|c|c|c|c|c|c|c|}
\hline $\begin{array}{l}\text { Modelo } \\
\end{array}$ & $x^{2}$ & $g l$ & $x^{2} / g l$ & $\Delta_{\chi^{2}}$ & $\Delta g l$ & CFI & TLI & IFI & SRMR & $\begin{array}{l}\text { RMSEA } \\
\text { (IC } 90 \%)\end{array}$ \\
\hline 1 & 31.03 & 18 & 1.72 & - & - & 0.94 & 0.90 & 0.94 & 0.055 & $\begin{array}{c}0.054 \\
(0.017- \\
0.085) \\
0.052\end{array}$ \\
\hline 2 & 38.40 & 23 & 1.67 & 7.37 & 5 & 0.93 & 0.90 & 0.93 & 0.065 & $\begin{array}{c}.0 .022 \\
(0.019- \\
0.0800) \\
0.049\end{array}$ \\
\hline 3 & 38.40 & 24 & 1.60 & 7.37 & 6 & 0.93 & 0.91 & 0.93 & 0.065 & $\begin{array}{l}(0.0 .015- \\
0.0777) \\
0.044\end{array}$ \\
\hline 4 & 44.42 & 30 & 1.48 & 13.39 & 12 & 0.93 & 0.93 & 0.93 & 0.063 & $\begin{array}{l}(0.008- \\
0.070)\end{array}$ \\
\hline
\end{tabular}

Nota: Modelo 1 = modelo sin restricciones; Modelo 2 = modelo con pesos de medida invariantes: Modelo 3 = modelo con covarianzas estructurales invariantes; Modelo $4=$ modelo con residuos de medida invariante. Fuente: elaboración propia.
Análisis de consistencia interna y estabilidad temporal

$\mathrm{El}$ análisis de consistencia interna reveló valores de $0.69(\alpha)$ y $0.70(\rho)$, mientras que el de estabilidad temporal (CCI) se situó en 0.80 (IC $95 \%=0.66,0.88)$.

\section{Discusión}

El objetivo de este estudio fue examinar las propiedades psicométricas del EAI en una muestra de estudiantes universitarios brasileños. Los resultados mostraron evidencias de validez factorial y aceptables valores de consistencia interna y estabilidad temporal, apoyando el uso del EAI para medir la adicción al ejercicio en el contexto brasileño. No obstante, los resultados del estudio plantean varias cuestiones que deberían ser tratadas en futuros trabajos.

En primer lugar, los resultados confirmaron la validez factorial del EAI. Todos los ítems correlacionaron significativamente con la medida global de adicción al ejercicio. El análisis factorial confirmatorio arrojó índices de ajuste aceptables para el modelo y una estabilidad temporal adecuada. No obstante, el análisis de consistencia interna arrojó valores justo en el límite usualmente aceptado de 0.70. Atendiendo a los resultados de anteriores trabajos realizados en distintos contextos culturales y que observaron la consistencia interna del instrumento, cabe destacar que estos informaron de la presencia tanto de valores adecuados (Sicilia, Alías-García, Ferriz, \& Moreno-Murcia, 2013; Terry et al., 2004) como por contra, inferiores a lo deseado (Griffiths et al., 2015). En tal sentido, los resultados de este estudio sugieren la existencia de una posible dualidad en los ítems que componen la escala. Aunque cada uno de los seis ítems que componen el EAI refleja un componente de la adicción (Griffiths, 1996), tres de los ítems (i. e., 1, 2 y 5) mostraron valores medios situados por debajo del punto medio de la escala, mientras que las medias de los tres restantes se situaron por encima de dicho punto. Esta distribución en 
las puntuaciones medias podría venir provocada por una interpretación diferenciada de los ítems. Así, cabe la posibilidad de que algunos estuviesen reflejando elementos asociados en mayor medida a consecuencias negativas (e. g., ha surgido conflicto con mi pareja o familia en relación con la cantidad de ejercicio que realizo). Por contra, otros podrían estar reflejando elementos asociados a consecuencias de carácter neutro o, incluso, de carácter positivo (e. g., con el tiempo he aumentado la cantidad de ejercicio que hago en una sesión). En esta línea, Johnston et al. (2011) pusieron de manifiesto que, en el contexto del ejercicio físico, las personas suelen resaltar más algunos componentes de la adicción que otros, percibiendo, no obstante, un continuo en el cual la línea que separa la conducta que podría ser calificada como normal de la adictiva no siempre está clara. Esto sugiere que tal vez algunas dimensiones definidas para la adicción conductual en el contexto del ejercicio físico no son percibidas de manera negativa de forma inequívoca. Por ejemplo, el componente tolerancia parece adoptar diferentes significados en el contexto del juego que en el ejercicio físico. Así, mientras incrementar el tamaño de la apuesta para experimentar el mismo efecto de euforia que inicialmente el jugador obtuvo con una apuesta mucho menor parece un claro componente adictivo, el hecho de aumentar la cantidad de ejercicio físico a consecuencia de la adaptación al entrenamiento no siempre puede ser entendido como una consecuencia negativa de esta conducta (Johnston et al., 2011). Futuros estudios deberían clarificar cómo son entendidos en el contexto específico del ejercicio físico los componentes de la adicción establecidos para un conjunto amplio de conductas.

En segundo lugar, el análisis multigrupo evidenció que, en línea con anteriores trabajos realizados en diversas culturas (Griffiths et al., 2015), la estructura del EAI permanecía invariante respecto del género. Este resultado resulta de especial interés atendiendo a anteriores trabajos que observaron diferencias en los niveles de adicción al ejercicio entre hombres y mujeres (González-Cutre \& Sicilia, 2012; Symons-Downs, Hausenblas, Davison, \&
Acharya, 2005; Weik \& Hale, 2009), ya que apoya el uso futuro de este instrumento en ambos grupos poblacionales. No obstante, futuros estudios deberían examinar si la estructura del EAI se mantiene invariante en grupos no considerados en el presente estudio. Por ejemplo, la investigación ha mostrado la existencia de una relación negativa entre la adicción al ejercicio y la edad (Allegre, Therme, \& Griffiths, 2007; Szabo et al., 1997). Por tanto, sería interesante comprobar si el EAI se muestra invariante en el contexto brasileño considerando diferentes grupos de edad.

Finalmente, de acuerdo con los valores de cribado del EAI, en este estudio se identificaron 16 estudiantes $(6.4 \%)$ en riesgo de adicción al ejercicio. Aunque la literatura indica datos muy dispares de prevalencia de adicción al ejercicio (Blaydon \& Lindner, 2002; Griffiths et al., 2005; Lejoyeux et al., 2008; SymonsDowns et al., 2004), los resultados de este estudio se muestran dentro de los límites (i. e., entre 3 y $15 \%$ ) informados en otros trabajos que utilizaron el EAI en jóvenes universitarios (Sicilia et al., 2013; Terry et al., 2004). Aunque la prevalencia de este fenómeno medida con el EAI entre universitarios no se muestra muy alta, los resultados obtenidos sugieren que un número importante de personas podrían precisar de ayuda. Por consiguiente, considerando las potenciales consecuencias negativas derivadas de la adicción al ejercicio, sería preciso profundizar en el estudio de los procesos que podrían propiciar este tipo de conducta y en la mejora de los procedimientos que permitirían su adecuada detección, prevención y tratamiento.

Aunque los resultados de este estudio proporcionan apoyo psicométrico al EAI, algunas limitaciones deben ser indicadas. En primer lugar, en este estudio, participaron mayoritariamente estudiantes universitarios de educación física y deportes, lo cual dificulta generalizar los resultados a otras poblaciones. En segundo lugar, no se aislaron otros desórdenes (e. g., trastornos de la alimentación) que la literatura ha identificado en ocasiones como concurrentes a la adicción al ejercicio (Freimuth et al., 2011). Por consiguiente, la posible presencia de este tipo de 
desórdenes podría haber contribuido a explicar el porcentaje de participantes clasificados en este estudio como en riesgo de adicción. Finalmente, aunque el EAI puede ser usado como un instrumento de cribado, un diagnóstico final de la adicción precisaría de exámenes médicos y entrevistas clínicas complementarias. En este sentido, los futuros estudios deberían verificar la sensibilidad del EAI en poblaciones clínicas.

En resumen, el EAI se presenta como una medida válida y fiable para evaluar la adicción al ejercicio en el contexto brasileño, aportando además evidencias de la validez transcultural del instrumento. El EAI se muestra como un instrumento práctico y de fácil aplicación, que puede permitir a los distintos profesionales involucrados en este ámbito realizar una primera valoración y cribado atendiendo a la presencia de síntomas de adicción al ejercicio.

\section{Referencias}

Allegre, B., Therme, P., \& Griffiths, M. D. (2007). Individual factors and the context of physical activity in exercise dependence: A prospective study of 'Ultra-marathoners'. International Journal of Mental Health and Addiction, 5(3), 233-243. http://dx.doi.org/ 10.1007/s11469-007-9081-9

American Psychiatric Association (1994). Diagnostic and statistical manual of mental disorders (4th ed.). Washington DC: APA.

American Psychiatric Association (2010). DSM-5 Development. Recuperado de http ://www.dsm5.org/Pages/Default.aspx

Blaydon, M. J., \& Lindner, K. J. (2002). Eating disorders and exercise dependence in triathletes. Eating Disorders, 10(1), 49-60.

Byrne, B. M. (2013). Structural equation modeling with AMOS: Basic concepts, applications, and programming. New York, NY: Routledge.

Carmack, M. A., \& Martens, R. (1979). Measuring commitment to running: A survey of runners' attitudes and mental states. Journal of Sport Psychology, 1(1), $25-42$.
Davis, C., Brewer, H., \& Ratusny, D. (1993). Behavioral frequency and psychological commitment: Necessary concepts in the study of excessive exercising. Journal of Behavioral Medicine, 16(6), 611-628. http://dx.doi.org/0160-7715/93/1 200-0611\$07.00/0

Farrell, J. M. \& Thompson, T. (1998). Exercise commitment and addiction: A fine line. Journal of Applied Recreation Research, 23(1), 63-75.

Ferguson, C. J. (2009). An effect size primer: A guide for clinicians and researchers. Professional Psychology: Research and Practice, 40(5), 532.

Fleiss, J. L. (2011). Design and analysis of clinical experiments. New York, NY: John Wiley \& Sons. http://dx.doi.org/10.1002/978111803 2923

Freimuth, M., Moniz, S., \& Kim, S. R. (2011). Clarifying exercise addiction: Differential diagnosis, co-occurring disorders, and phases of addiction. International Journal of Environmental Research and Public Health, 8(10), 4069-4081. http://dx.doi.org/10.339 0/ijerph8104069

Glasser, W. (1976). Positive addiction. New York, NY: Harper y Row.

González-Cutre, D. \& Sicilia, A. (2012). Dependencia del ejercicio físico en usuarios españoles de centros de acondicionamiento físico (fitness): Diferencias según el sexo, la edad y las actividades practicadas. Behavioral Psychology/Psicología Conductual, 20(2), 349-364.

Grant, J. E., Potenza, M. N., Weinstein, A., \& Gorelick, D. A. (2010). Introduction to Behavioral Addictions. The American Journal of Drug and Alcohol Abuse, 36(5), 233-241. http://dx.doi.org/10.3109/009529 90.2010 .491884

Griffiths, M. D. (1996). Behavioural addiction: An issue for everybody? Employee Counselling Today, 8(3), 19-25. http://dx.do i.org/10.1108/13665629610116872

Griffiths, M. D., Szabo, A. \& Terry, A. (2005). The exercise addiction inventory: a quick and easy screening tool for 
health practitioners. British Journal of Sports Medicine 39(6), e30. http://dx.doi.org/10.1 136/bjsm.2004.017020

Griffiths, M. D., Urbán, R., Demetrovics, Z., Lichtenstein, M. B., de la Vega, R., Kun, B., ... \& Szabo, A. (2015). A cross-cultural re-evaluation of the Exercise Addiction Inventory (EAI) in five countries. Sports Medicine-Open, 1(1), 1-5.

Hailey, B. J. \& Bailey, L. A. (1982). Negative addiction in runners: A quantitative approach. Journal of Sport Behavior, 5(3), 150-154.

Hambleton, R. K. \& Patsula, L. (1998). Adapting tests for use in multiple languages and cultures. Social Indicators Research, 45(1-3), 153-171.

Hausenblas, H. A. \& Symons-Downs, D. (2002a). Exercise Dependence Scale-21 Manual. Gainesville, FL: Florida University.

Hausenblas, H. A. \& Symons-Downs, D. (2002b). Exercise dependence: A systematic review. Psychology of Sport and Exercise, 3(2), 89-123. http://dx.doi.org/10. 1016/S1469-0292(00)00015-7

Hausenblas, H. A. \& Symons-Downs, D. (2002c). How much is too much? The development and validation of the Exercise Dependence Scale. Psychology y Health, 17(4), 387-404. http://dx.doi.org/10.1080/ 0887044022000004894

Hu, L. \& Bentler, P. M. (1999). Cutoff criteria for fit indexes in covariance structure analysis: Conventional criteria versus new alternatives. Structural Equation Modeling, 6, 1-55. http://dx.doi.org/10.1080/1070551 9909540118

Johnston, O., Reilly, J., \& Kremer, J. (2011). Excessive exercise: From quantitative categorisation to a qualitative continuum approach. European Eating Disorders Review, 19, 237-248. http://dx.doi.org/10.1002/ erv. 970

Kagan, D. M. (1987). Addictive personality factors. The Journal of Psychology, 121(6), 533-538. http://dx.doi.org/10.1080/002239 80.1987 .9712681
Lejoyeux, M., Avril, M., Richoux, C., Embouazza, H., \& Nivoli, F. (2008). Prevalence of exercise dependence and other behavioral addictions among clients of a Parisian fitness room. Comprehensive Psychiatry, 49(4), 353-358. http://dx.doi.org/10.1016/j .comppsych.2007.12.005

Lynn, M. R. (1986). Determination and quantification of content validity. Nursing Research, 35, 382-385.

Mónok, K., Berczik, K., Urbán, R., Szabo, A., Griffiths, M. D., Farkas, J., ... \& Demetrovics, Z. (2012). Psychometric properties and concurrent validity of two exercise addiction measures: A population wide study. Psychology of Sport and Exercise, 13(6), 739-746. http://dx.doi.org/10.1016/j .psychsport.2012.06.003

Morgan, W. P. (1979). Negative addiction in runners. The Physician and SportsMedicine, 7, 57-77.

Nunnally, J. C. \& Bernstein, I. J. (1995). Teoría psicométrica. Madrid: McGraw-Hill.

Ogden, J., Veale, D., \& Summers, Z. (1997). The development and validation of the Exercise Dependence Questionnaire. Addiction Research EO Theory, 5(4), 343-356.

Raykov, T. (2004). Behavioral scale reliability and measurement invariance evaluation using latent variable modeling. Behavior Therapy, 35, 299-331. http://dx.doi.org/10.1016/S00 05-7894(04)80041-8

Sicilia, A., Alías-García, A., Ferriz, R., \& Moreno-Murcia, J. A. (2013). Spanish adaptation and validation of the Exercise Addiction Inventory (EAI). Psicothema, 25(3), 377-383. http://dx.doi.org/10.7334/ psicothema2013.21

Sussman, S., Lisha, N., \& Griffiths, M. D. (2011). Prevalence of the addictions: A problem of the majority or the minority? Evaluation $\mathbb{E}$ the Health Professions, 34(1), 3-56. http://dx .doi.org/10.1177/0163278710380124

Symons-Downs, D., Hausenblas, H. A., Davison, K. K., \& Acharya, J. (2005). Ethnocultural and gender differences in exercise dependence symptoms. Journal of 
Sport and Exercise Psychology, 27, S150. S151.

Symons-Downs, D., Hausenblas, H. A., \& Nigg, C. R. (2004). Factorial validity and psychometric examination of the Exercise Dependence Scale-Revised. Measurement in Physical Education and Exercise Science, 8(4), 183-201. http://dx.doi.org/10.1207/s1 5327841mpee0804_1

Szabo, A., Frenkl, R., \& Caputo, A. (1997). Relationship between addiction to running, commitment, and deprivation from running: A study on the internet. European Yearbook of Sport Psychology, 1, 130-147.

Terry, A., Szabo, A., \& Griffiths, M. D. (2004). The exercise addiction inventory: a new brief screening tool. Addiction Research and Theory, 12(5), 489-499. http://doi.org/10.1 080/16066350310001637363

Weik, M., \& Hale, B. D. (2009). Contrasting gender differences on two measures of exercise dependence. British Journal of Sports Medicine, 43(3), 204-207. http://doi. org/10.1136/bjsm.2007.045138

\section{Versión Brasileña del Exercise Addiction Inventory (EAI)}

\footnotetext{
1. O exercício físico é a coisa mais importante de minha vida.

2. Tem surgido conflitos com meu/minha companheiro/a e/ou minha família em

relação à quantidade de atividade física que realizo.

3. Uso o exercício físico como uma forma de mudar meu estado de ânimo.

4. Com o tempo tenho aumentado a quantidade de exercício que faço em cada

sessão.

5. Se preciso faltar a uma sessão de atividade física fico mal humorado e irritado.

6. Se deixo de fazer exercício e depois começo novamente, sempre tento fazer

tanto exercício como fazia anteriormente.
}

\section{Notas}

* Artículo de investigación 